\title{
Propuesta para la inteǵración de la Norma CWA 15793:2008 a las normas NTC-ISO 9001:2008, NTC-ISO 17025:2005 y NTC OHSAS 18001:2007 en el Laboratorio Nacional de Diagnóstico Veterinario del Instituto Colombiano Agropecuario (ICA)*
}

Integration of the standards CWA 15793:2008, NTC-ISO 9001:2008, NTC-ISO 17025:2005 and NTC OHSAS 18001:2007 in the National Veterinary Diagnostic Laboratory of the Colombian Agricultural and Livestock Institute (ICA)

Inteǵração das normas CWA 15793: 2008, NTC-ISO 9001: 2008, NTCISO 17025: 2005 e NTC OHSAS 18001: 2007 no Laboratório Nacional de Diagnóstico Veterinário do Instituto Colombiano agropecuário (ICA)

Recibido: 5 de mayo de 2017 Revisado: 15 de octubre de 2017 Aceptado: 20 de diciembre de 2017

Alba Bernarda Porras Santiago*

Laboratorio Nacional de Diagnóstico Veterinario del Instituto Colombiano Agropecuario

Artículo de resultado de investigación. DOI: http://dx.doi.org/10.15332/s2145-1389.2018.0001.05

** Magíster en Calidad y Gestión Integral. Bacterióloga y laboratorista clínica. Instituto Colombiano Agropecuario (ICA). Correo electrónico: albaporras@hotmail.com, alba.porras@ica.gov.co 


\section{RESUMEN}

Los laboratorios de diagnóstico veterinario son los encargados de la detección de enfermedades en animales tanto domésticos como silvestres en las diferentes partes del mundo, por medio de la realización de distintas pruebas de laboratorio encaminadas tanto a la vigilancia como a la detección de las enfermedades (salud y producción animal), lo que ha permitido el comercio internacional de estos productos. Atendiendo a ello, los laboratorios de diagnóstico veterinario deben garantizar la fiabilidad de sus resultados, para lo cual han adoptado modelos de gestión en busca de resultados aceptados en el contexto internacional, y de esta manera favorecer el intercambio comercial de animales y sus productos. Para ello, se realizó una investigación de tipo mixta, cuyo objetivo fue elaborar una propuesta de integración de la norma CWA 15793:2008, la NTC-ISO 9001:2008, la NTC-ISO 17025:2005 y la NTC OHSAS 18001:2007. Para ello, en primer lugar se elaboró una matriz de correlación de requisitos de las normas de interés, en segundo lugar, con la intención de conocer cómo se estaba trabajando en el laboratorio objeto de investigación la gestión del riesgo biológico, se elaboró una lista de chequeo basada en los requerimientos de la norma, y finalmente con el objetivo de tener un mayor conocimiento del problema, se recurrió a la conformación de un grupo focal conformado por expertos en temas de manejo de riesgo biológico, manejo de sistemas de gestión, planeación y laboratorio, con los que se analizó la información obtenida mediante la lista de chequeo. Se logró plantear una propuesta de integración de las normas internacionales mencionadas con la norma para gestión del bioriesgo teniendo en cuenta los lineamientos dados por la Organización Mundial de Salud Animal (OIE) y adoptados en la Asamblea Mundial en mayo de 2015; además, complementa las medidas ya adoptadas en el laboratorio analizado y proporciona herramientas muy valiosas para el trabajo adecuado dentro de las instalaciones, teniendo en cuenta el ambiente y la comunidad circundante.

Palabras clave: laboratorio de diagnóstico veterinario, gestión del bioriesgo, integración.

\section{ABSTRACT}

Veterinary diagnostic laboratories are responsible for the detection of diseases in both domestic and wild animals in different parts of the world, by conducting different laboratory tests aimed at both surveillance and detection of different diseases (health and animal production), which has allowed the international trade of these products. In view of this, veterinary diagnostic laboratories must guarantee the reliability of their results, for which they have adopted management models in search of results accepted in the international context, and thus favor the commercial exchange of animals and their products. For this, a mixed type research was carried out whose objective was to elaborate a proposal of integration of the standard CWA 15793:2008, the NTC-ISO 9001:2008, the NTC-ISO 17025:2005 and NTG OHSAS 18001:2007. To do this, first a correlation matrix of requirements of the standards of interest was prepared, secondly with the intention of knowing how the biological risk management was being worked in the laboratory under investigation, a checklist was made based on the requirements of the standard and finally with the aim of having a better knowledge of the problem, a focus group was formed consisting of experts in biological risk management issues, handling of management systems, planning and laboratory with which the information obtained through the checklist was analyzed. It was possible to make a proposal for the integration of the aforementioned international standards with the biorisk management standard, taking into account the 
guidelines given by the World Organization for Animal Health (OIE) and adopted at the World Assembly in May 2015, it also complements the measures already adopted in the analyzed laboratory and provides very valuable tools for proper work within the facilities, taking into account the environment and the surrounding community.

Keywords: Veterinary diagnostic laboratory, biorisk management, integration.

\section{RESUMO}

Os laboratórios de diagnóstico veterinário são os responsáveis da detecção de doenças em animais domésticos e selvagens nas diferentes partes do mundo, por meio da realização de diferentes testes laboratoriais destinados à vigilância e à detecção das diferentes doenças (saúde e produção animal), o que permitiu o comércio internacional destes produtos. Atendendo a tudo isto, os laboratórios de diagnóstico veterinário devem garantir à fiabilidade de dois seus resultados, para o qual adotaram modelos de gestão em procura de resultados aceitos no contexto internacional, e desta maneira favorecer o intercâmbio comercial de animais e seus produtos. Para isso, foi realizada uma pesquisa mista, cujo objetivo foi elaborar uma proposta de integração da norma CWA 15793:2008, a NTC-ISO 9001:2008, a NTC-ISO 17025:2005 e a NTC OHSAS 18001:2007. Por conseguinte, em primeiro lugar foi elaborada uma matriz de correlação de requisitos das normas de interesse, em segundo lugar com a intenção de conhecer como se estava trabalhando no laboratório objeto da investigação, a gestão de riscos biológicos, foi elaborado uma Lista de verificação baseada nos requerimentos da norma e finalmente com o objetivo de ter um maior conhecimento do problema, se formou um grupo focal conformado por especialistas em temas de manejo de risco biológico, manejo de sistemas de gestão, planejamento e laboratório com os quais se analisou a informação resultante da lista de verificação. Foi possível apresentar uma proposta de integração das normas internacionais acima mencionadas com a norma de gestão de risco biológico, tendo em conta os lineamentos da Organização Mundial da Saúde Animal (OIE) e adotadas na Assembleia Mundial em maio de 2015, além disso complementa as medidas. Já adotadas no laboratório analisado e proporciona ferramentas muito valiosas para 0 trabalho adequado dentro das instalações, tendo em conta 0 ambiente e a comunidade circundante.

Palavras-chave: laboratório de diagnóstico veterinário, gestão de risco biológico, integração.

\section{INTRODUCCIÓN}

El presente trabajo busca generar una propuesta de integración de la gestión del riesgo en el Laboratorio Nacional de Diagnóstico Veterinario en concordancia con las directrices de la Organización Mundial de Sanidad Animal (OIE), teniendo en cuenta que el Laboratorio Nacional de Diagnóstico Veterinario (LNDV) como parte del ente regulador en esta materia a nivel nacional y que de su desempeño sobresaliente dependen no solo la salud animal, sino también la vigilancia de fronteras y el diagnóstico y vigilancia que permite al país el intercambio de productos con otros países, debe estar a la vanguardia de la adopción y puesta en marcha de las normas que regulan el buen desempeño de los laboratorios de diagnóstico veterinario, donde se presenta la necesidad de integrar la norma de la gestión del bioriesgo como complemento a las normas que ya tienen implementadas, según lo descrito en el capítulo 1.1.4 del Manual terrestre de la OIE (2015). 
La integración de la gestión del riesgo biológico en los laboratorios del LNDV servirá de complemento a los sistemas que ya se encuentran al interior de la organización, como resultado se obtendrán beneficios que otras empresas a nivel mundial han logrado. En diferentes investigaciones se han identificado beneficios desde el punto de vista administrativo que se pueden obtener con la implementación de sistemas de gestión: minimización de documentación y registros, menos burocracia y reducción de papeleo, ahorro de costos por optimización de tiempo y recursos, simplificación de auditorías internas y externas, y desde el punto de vista de la compresión de los procesos genéricos, más interrelaciones o sinergias entre los diferentes sistemas, objetivos y metas coordinadas y equilibradas y organización y responsabilidades concentradas. Es por lo tanto importante que los laboratorios conozcan, se familiaricen y se les brinde un soporte adecuado para la integración de los diferentes sistemas de gestión, como lo menciona Heras, Bernardo y Casadesús (2007):

Con todo, existe un grado de consenso muy elevado al referirse a la importancia de que los nuevos estándares pertenecientes a ámbitos diferentes de gestión se puedan integrar fácilmente en un único sistema integrado de gestión, ya que, de lo contrario, la implementación y certificación aislada de los diferentes sistemas de gestión redundaría de forma negativa en la capacidad competitiva de las empresas. (p. 172)

Teniendo presente que las empresas cuentan con diferentes procesos, en los cuales también funcionan de manera integrada para poder generar el producto 0 servicio.

Con respecto a las razones mencionadas en el caso de los laboratorios de diagnóstico veterinario, y aprovechando la sinergia de los sistemas a integrar y su afinidad con el trabajo en los laboratorios de diagnóstico veterinario, en especial de las norma de gestión del riesgo biológico y la de requisitos generales relativos a la competencia de los laboratorios de ensayo y calibración, se propone la integración de los sistemas de gestión, esperando que se vea reflejado en procesos más eficientes, con una significativa disminución de esfuerzos y desempeño sobresaliente en los laboratorios.

Como ya se mencionó, los laboratorios de diagnóstico veterinario se encuentran regulados por la Organización Mundial de Sanidad Animal (OIE), la cual vela por la fiabilidad de las pruebas realizadas en estos, para ello reconoce la necesidad de contar con normas de gestión reconocidas internacionalmente, para tal fin la comisión de normas biológicas de la OIE referencia la norma ISO/IEG 17025:2005, la ISO 9001:2008 y a partir del 2014 la norma del Comité Europeo de Normalización CWA 15793 para estandarizar los procedimientos en los laboratorios de diagnóstico a nivel mundial, y facilitar los intercambios comerciales y de información en este tipo de laboratorios, sin mencionar que deben estar integrados a pesar de ello por motivos de eficiencia de los recursos y la necesidad de disminuir procesos burocráticos dentro de las instituciones. Para aumentar su eficiencia se propone la integración de estos en el presente estudio con la finalidad de favorecer no solo al LNDV sino también que pueda ser utilizada por otros laboratorios de este tipo.

Teniendo presente que los sistemas de gestión van evolucionando a través del tiempo de acuerdo a las necesidades de la industria, debido al proceso de cambio que enfrenta actualmente la economía mundial, como también a eventos fortuitos permitiendo que las organizaciones los utilicen de acuerdo a las condiciones particulares, en el caso de los laboratorios de diagnóstico veterinario para satisfacer sus necesidades, encontramos sistemas como el de gestión del riesgo biológico en el laboratorio y otros que disminuyen la posibilidad de la ocurrencia de eventos que afecten adversamente el cumplimiento de los objetivos, lo que se traduce en pérdidas y grave amenaza para la sanidad animal y alimentaria (Simbaqueba, 2015). 
Tenemos casos como la Unión Europea en los que la responsabilidad de la sanidad es de cada uno de los países miembros por medio de sus oficinas veterinarias y alimentarias, las cuales se encuentran agrupadas la mayoría en la Dirección General de Sanidad y Política de Consumidores (DG SANCO), que es la responsable de la normativa como de la inspección y aplicación de ella por los países miembros. La evaluación del riesgo en materias veterinarias es responsabilidad de la Autoridad Europea de Seguridad Alimentaria EFSA (2002), una de las 23 agencias comunitarias en las cuales también se realiza acompañamiento y regulación del diagnóstico veterinario.

El riesgo biológico en los laboratorios es reconocido, dado que en los laboratorios se presenta una serie de riesgos de origen y consecuencias muy variadas, relacionados básicamente con las instalaciones, los productos que se manipulan y las operaciones que se realizan con ellos, además asocia la gestión de calidad en los laboratorios a un elevado nivel de seguridad, la prevención de los riesgos en el laboratorio presenta unas características propias que la diferencian de otras áreas productivas; la norma CWA 15793:2008, norma europea de gestión de riesgo biológico en el laboratorio, está basada en un sistema de gestión implicando un sistema de procesos que buscan la mejora, la efectividad y eficiencia de la organización.

En países latinoamericanos donde encontramos organismos con características muy similares a las del Instituto Colombiano Agropecuario (ICA), dedicados a salvaguardar la sanidad animal como los SENASA México, SENASA Honduras, SENASA Argentina, SENASA Perú; todos ellos con políticas de calidad encaminadas a dar cumplimiento a los requisitos de la OIE, destacándose la adopción de normas como la ISO 9000 e ISO 17025 dentro de las cuales es considerada la gestión del riesgo biológico, y a partir del año 2014 también se incluyó la norma CWA 15793. Todas con la misma necesidad de adoptar la norma de gestión de riesgo biológico.

En el año 2010, la Superintendencia de Salud solicitó a los servicios de gobierno la identificación de por lo menos 20 riesgos de gran criticidad para el logro de objetivos, que debe estar acompañada de un plan de tratamiento que incluya su manejo y administración. Dentro de los cuales muy posiblemente se encontrarán los riesgos asociados a la sanidad animal que están estrechamente relacionados con muchas de las actividades del comercio que representan un tema de gran interés para los gobiernos.

Por todas estas razones que se han mencionado, la integración de la gestión del riesgo biológico en los laboratorios del LNDV servirá de complemento a los sistemas que ya se encuentran al interior de la organización, además se obtendrán beneficios que otras empresas a nivel mundial han logrado, como lo establece Jorgesen (2008) en su investigación realizada en Dinamarca, identificando los beneficios desde el punto de vista administrativo que se pueden obtener: minimización de documentación y registros, menos burocracia y reducción de papeleo, ahorro de costos por optimización de tiempo y recursos, simplificación de auditorías internas y externas; y desde el punto de vista de la compresión de los procesos genéricos: más interrelaciones o sinergias entre los diferentes sistemas, objetivos, metas coordinadas y equilibradas, organización y responsabilidades concentradas. Es por lo tanto importante que los laboratorios conozcan, se familiaricen y se les brinde un soporte adecuado para la integración de los diferentes sistemas de gestión.

\section{METODOLOGÍA}

La investigación realizada de acuerdo a sus características es de tipo mixto, pues se integran un método 
cuantitativo y uno cualitativo, a fin de obtener una visión más clara del fenómeno, permitiendo complementar la información obtenida en la utilización de uno de los métodos con el apoyo del segundo método utilizado para la recolección de la información, lo que nos permite realizar un abordaje más integral del fenómeno y considerar una visión completa, teniendo en cuenta que se llevó a cabo en un grupo específico de la población (Hernández, Fernández y Baptista, 2014).

Para el desarrollo de la investigación, después de la recolección de la información, se elaboró una matriz con las normas de interés en el estudio, para identificar los requisitos comunes a todas, como es bien sabido, la estructura de las normas es muy parecida. Se encontraron varios puntos de relación, en los que se deben unificar actividades, posteriormente con la intención de conocer cómo se estaba trabajando en los laboratorios del LNDV la gestión del riesgo biológico, se elaboró una lista de chequeo basada en los requerimientos de la norma; finalmente con el objetivo de profundizar más y tener un mayor conocimiento del problema se recurrió a la conformación de un grupo focal conformado por expertos en temas de manejo de riesgo biológico, manejo de sistemas de gestión, planeación y laboratorio con los que se analizó la información obtenida mediante la listas de chequeo.

Tabla 1. Técnicas para la recolección de la información

\begin{tabular}{|c|c|c|}
\hline Objetivo & $\begin{array}{l}\text { Técnicas para la recolección de la } \\
\text { información }\end{array}$ & $\begin{array}{l}\text { Técnicas para el procesamiento de la } \\
\text { información }\end{array}$ \\
\hline $\begin{array}{l}\text { Identificar los aspectos comunes de los } \\
\text { sistemas de gestión a integrar NTC ISO } \\
\text { 9001, ISO/IEC 17025, OHSAS } 18001 \text { y } \\
\text { CWA } 15793 \text { en el LNDV ICA. }\end{array}$ & $\begin{array}{l}\text { Se partió de la matriz de correlación de los } \\
\text { requisitos documentales de las normas, lo cual } \\
\text { sirvió para ver que estas son compatibles. }\end{array}$ & $\begin{array}{l}\text { Se realizó una comparación de los requisitos } \\
\text { de las cuatro normas para identificar los } \\
\text { elementos comunes. }\end{array}$ \\
\hline \multirow{2}{*}{$\begin{array}{l}\text { Identificar el estado de la gestión del } \\
\text { riesgo biológico en los laboratorios del } \\
\text { LNDV ICA. }\end{array}$} & $\begin{array}{l}\text { Elaboración y aplicación de listas de chequeo, } \\
\text { basadas en los requisitos de la norma de } \\
\text { gestión del riesgo biológico CWA } 15793 \text {. }\end{array}$ & \multirow{2}{*}{$\begin{array}{l}\text { Análisis estadístico de los porcentajes de } \\
\text { respuesta obtenidos. } \\
\text { Obtención de información de expertos. }\end{array}$} \\
\hline & $\begin{array}{l}\text { Conformación de un grupo focal para analizar } \\
\text { los resultados obtenidos en el laboratorio } \\
\text { y determinar la manera de proponer la } \\
\text { integración. }\end{array}$ & \\
\hline $\begin{array}{l}\text { Estructurar una propuesta en la que se } \\
\text { contemplen los aspectos que se deben } \\
\text { tener en cuenta para integrar la gestión } \\
\text { del riesgo biológico en los laboratorios } \\
\text { LNDV ICA. }\end{array}$ & $\begin{array}{l}\text { Basado en los requerimientos y teniendo } \\
\text { en cuenta lo que está implementado de las } \\
\text { normas ISO 9001, ISO/IEC } 17025 \text { y OHSAS } \\
18000 .\end{array}$ & $\begin{array}{l}\text { La propuesta sugiere los lineamientos que } \\
\text { se deben seguir para la integración de la } \\
\text { norma CWA } 15793 \text { sobre gestión del riesgo } \\
\text { biológico. }\end{array}$ \\
\hline
\end{tabular}

Fuente: elaboración propia. 


\section{RESULTADOS Y DISCUSIÓN}

Como resultado del análisis de las listas de chequeo, se obtuvo una visión de la gestión del bioriesgo en el LNDV, desde la óptica de los profesionales encargados del desarrollo de las actividades de análisis que involucran trabajo con agentes biológicos, se muestran los enunciados con mayores puntajes de cumplimiento, así como los que obtuvieron las más bajas puntuaciones con respecto al cumplimiento o grado de conocimiento por parte del personal. A continuación, se muestran estos enunciado como fortalezas en figura 1 y como debilidades en la figura 2.
Se observa que se tienen implementados y son conocidos por el personal los requerimientos de la norma que hacen referencia a aspectos relacionados con la competencia del personal, las buenas prácticas de laboratorio, inactivación de agente biológicos y toxinas, entre otros; por ser estos puntos que se han trabajado como parte de la implementación de normas como la ISO 17025:2005, ISO 9001:2008 y OSHAS 18001:2007, las cuales se encuentran integradas en los diferentes procesos del laboratorio y pueden ser los puntos de articulación con la norma CWA 15793.

Figura 1. Fortalezas de la gestión del riesgo biológico en el LNDV

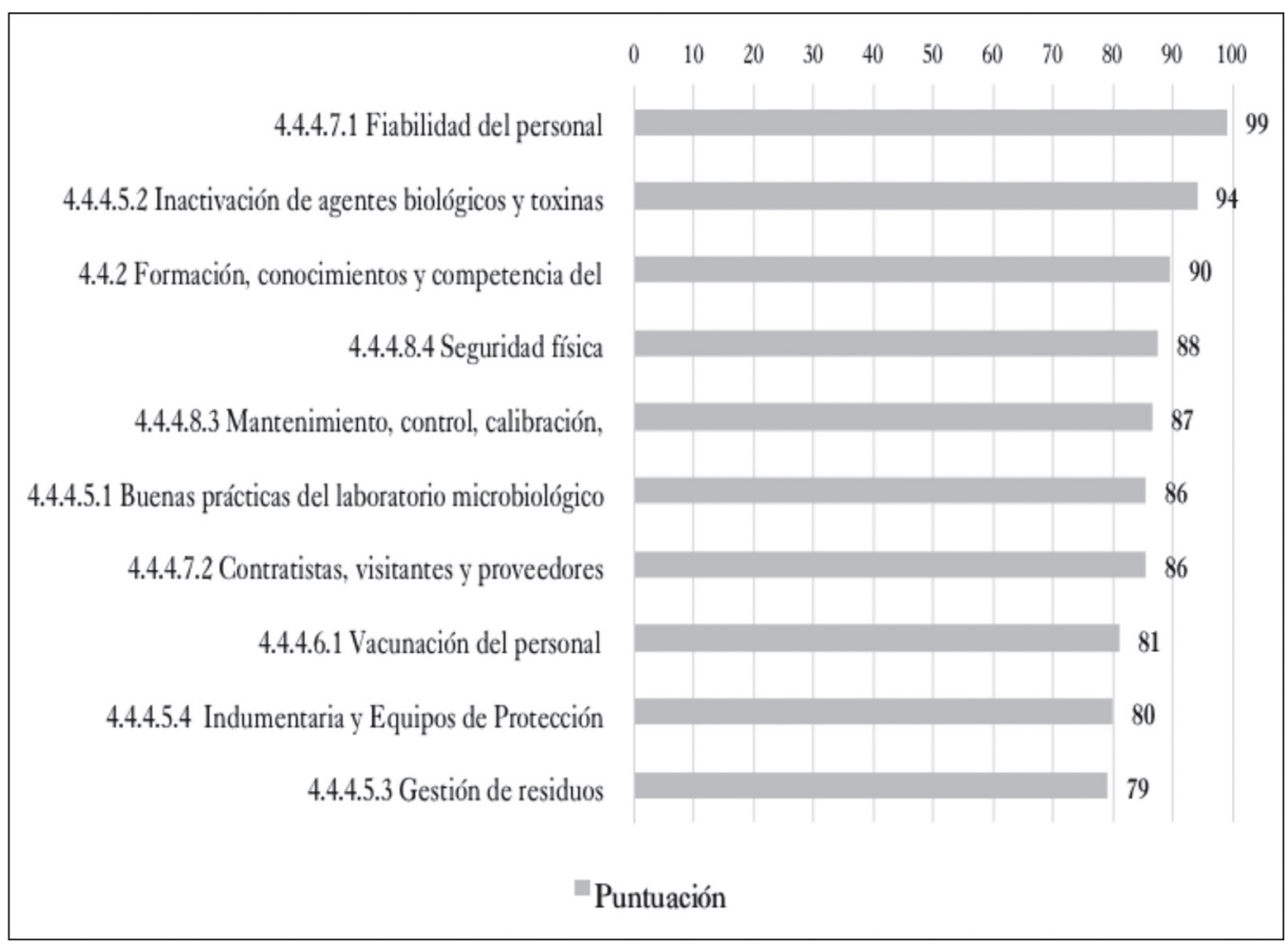

Fuente: elaboración propia. 
Figura 2. Debilidades de la gestión del bioriesgo en el LNDV

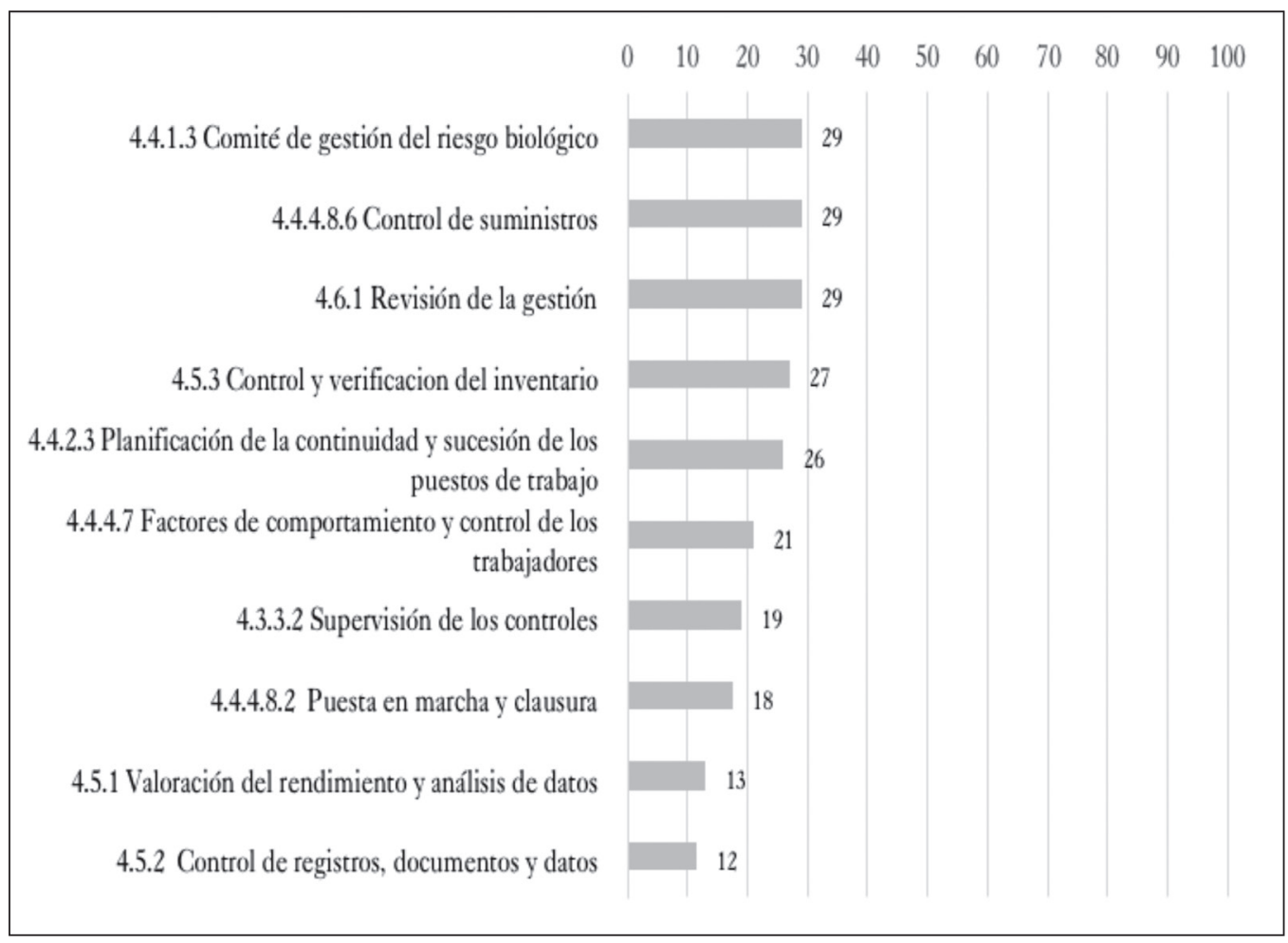

Fuente: elaboración propia.

Teniendo en cuenta que los sistemas de gestión se encuentran ya integrados en la institución, y que el objetivo de la propuesta es integrar la gestión de bioriesgo a estos sistemas ya integrados, los análisis de las listas de chequeo nos presentan puntos que deben ser trabajados de manera especial para lograr esta integración.

Con la ayuda del grupo focal se estableció que se deben seguir los siguientes pasos para integrar la gestión de bioriesgo:

- Sensibilización del personal mediante charlas y talleres.

- Capacitación en el sistema de gestión del bioriesgo, donde se explique que "el análisis del riesgo biológico incluye la detección de peligros biológicos, una evaluación del laboratorio, seguida de la gestión de los riesgos biológicos relacionados, y una comunicación del riesgo biológico" OIE (2015).

- Lograr el compromiso de los directivos.

- Designar los líderes y responsables de la integración, asignándoles las funciones y autorizaciones necesarias.

- Incluir en el alcance del sistema de gestión integrado teniendo en cuenta que la norma ISO 17025:2005 y la norma CWA 15793:2008 son exclusivas de los laboratorios.

- Ajustar las políticas para integrar en estas la gestión del riesgo biológico.

- Rediseñar los objetivos alineándolos con las nuevas políticas. 
- Establecer controles e indicadores para poder medir el desempeño del sistema integrado.

- Capacitar a los funciones y niveles necesarios, como por ejemplo, al grupo del comité de gestión del bioriesgo, al grupo de auditores para dar un nuevo enfoque a estos procesos.

- Establecer el mapa de procesos del Laboratorio Nacional de Diagnóstico Veterinario, con el fin de determinar el grado de integración en cada uno de ellos y hacer los ajustes necesarios para la integración de la gestión del bioriesgo, ya que un método conformado por el mapa de procesos, el análisis de elementos comunes y el ciclo PHVA, parecen ayudar a obtener los mejores resultados para una integración total de un sistema (Bernardo, Simon, Taríy Molina, 2015).

- Establecer los indicadores de cumplimiento en cada uno de los procesos para poder hacer un seguimiento a su desempeño.

- Realizar el análisis de riesgo en los procesos identificados teniendo en cuenta los riesgos de tipo biológico.

\section{CONCLUSIONES}

La gestión del bioriesgo es un tema en el que se ha trabajado en los laboratorios del LNDV mediante la adopción de las buenas prácticas de laboratorio, a pesar de esto es importante que se sigan unos lineamientos que conduzcan a la mejora y permitan la integración adecuada de la gestión, como lo recomienda la OIE en su Manual terrestre. La adopción de una estrategia de análisis del riesgo para la gestión de los riesgos biológicos con fines de bioseguridad y bioprotección en los laboratorios veterinarios y en las instalaciones de los animales, proporciona a los países miembros un medio de adaptación de sus políticas y procedimientos nacionales de sanidad animal relativos a los laboratorios, a sus particulares circunstancias y a sus prioridades del bioriesgo como un sistema de gestión con todas las característica y el cumplimiento de los OIE (2015).

En el momento actual que atraviesa la institución, el hecho de tener ya implementados sistemas de gestión de la calidad ISO 9001:2008 y la de requisitos generales para la competencia de los laboratorios de ensayo y calibración ISO 17025:2005 que están ya integrados en los procesos, y teniendo en cuenta que la institución se encuentra en el proceso de adopción de la norma OHSAS 18001:2007 para gestión de seguridad y salud en el trabajo, el cual tiene una alta sinergia con la gestión del bioriesgo CWA 15793, se debe aprovechar para unificarlos en un sistema integrado de gestión que realmente disminuya la documentación y optimice los recursos, además de poder optimizar los proceso de auditoría.

Es importante resaltar que la integración de la gestión del riesgo biológico tiene un alto impacto, pues la bioseguridad del laboratorio es el conjunto de principios y prácticas destinados a prevenir el escape no intencionado o la exposición accidental a agentes o toxinas biológicos. La bioprotección del laboratorio, por su parte, es el control físico de los agentes o toxinas biológicos dentro del laboratorio, que tiene por objetivo prevenir su pérdida, robo, uso indebido y acceso no autorizado o liberación intencionada no OIE (2015) autorizada.

\section{REFERENCIAS}

Autoridad Europea de Seguridad Alimentaria-EFSA. (2002). Visión general. Recuperado de https://europa.eu/european-union/about-eu/agencies/efsa_es

Bernardo, M., Simon, A., Tarí, J., y Molinea, J. (2015). Benefits of management systems integration: a 
literature review. Journal of Cleaner Production, 94(1), 260-267.

Heras, I., Bernardo, M., y Casadesús, M. (2007). La integración de sistemas de gestión basados en estándares internacionales: Resultados de un estudio empírico realizado en la CAPV. Revista de Dirección y Administración de Empresas, 14, 155-174.

Hernández, R., Fernández, C., y Baptista, P. (2014). Metodología de la investigación, (6. ${ }^{\mathrm{a}}$ ed.). México D. F.: McGraw-Hill.

Jorgensen, T. (2008). Towards more sustainable management systems: through life cycle management and integration. Journal of Cleaner Porduction, 16(10), 1071-1080. Retrieved from https://doi. org/10.1016/j.jclepro.2007.06.006

OIE. (2015). Manual de las pruebas de diagnóstico y de las vacunas para los animales terrestres (mamiferos, aves y abejass. París, Francia: Office International des Epizooties. Recuperado de http://www.oie. int/doc/ged/d6508.pdf

Simbaqueba, L. (2015). Importancia de la gestión integral de riesgo. Recuperado de https://incp.org.co/Site/ info/archivos/riesgo.pdf 